\title{
Seroepidemiology of Entamoeba histolytica Infection in General Population in Rural Durango, Mexico
}

\author{
Cosme Alvarado-Esquivel ${ }^{\mathrm{a}, \mathrm{c}}$, Jesus Hernandez-Tinoco ${ }^{\mathrm{b}}$, Luis Francisco Sanchez-Anguiano ${ }^{\mathrm{b}}$
}

\begin{abstract}
Background: The seroepidemiology of Entamoeba histolytica in Mexico has been scantily studied. The aim of the study was to determine the seroprevalence and correlates of E. histolytica antibodies in adults in rural areas in Durango, Mexico.

Methods: Through a cross-sectional study, E. histolytica IgG antibodies were determined in 282 adults living in rural Durango, Mexico using an enzyme-linked immunoassay. In addition, seroprevalence association with the socio-demographic, housing conditions, and behavioral characteristics of the subjects studied was investigated.
\end{abstract}

Results: One hundred and eighteen $(41.8 \%)$ of the 282 rural subjects had anti-E. histolytica IgG antibodies. Multivariate analysis showed that $E$. histolytica exposure was positively associated with source of drinking water $(\mathrm{OR}=2.73 ; 95 \% \mathrm{CI}: 1.33-5.58 ; \mathrm{P}=0.005)$, and poor education of the head of the family $(\mathrm{OR}=1.53 ; 95 \% \mathrm{CI}: 1.03-2.27$; $\mathrm{P}=0.03$ ). In contrast, $E$. histolytica exposure was negatively associated with consumption of unpasteurized cow milk (OR $=0.55 ; 95 \%$ CI: $0.31-0.96 ; \mathrm{P}=0.03)$, and crowding at home $(\mathrm{OR}=0.33 ; 95 \% \mathrm{CI}$ : $0.17-0.64 ; \mathrm{P}=0.0009)$.

Conclusions: The seroprevalence of E. histolytica infection found in adults in rural Durango is high compared with those reported in other Mexican populations. The correlates of E. histolytica seropositivity found in the present study may be useful for the planning of optimal preventive measures against $E$. histolytica infection.

Keywords: Entamoeba histolytica; Seroepidemiologic studies; Rural population; Risk factors; Mexico

\footnotetext{
Manuscript accepted for publication March 16, 2015

aBiomedical Research Laboratory, Faculty of Medicine and Nutrition, Juarez University of Durango State, Avenida Universidad S/N, 34000 Durango, Mexico

bInstitute for Scientific Research "Dr. Roberto Rivera-Damm", Juarez University of Durango State, Avenida Universidad S/N, 34000 Durango, Mexico ${ }^{\mathrm{c}}$ Corresponding Author: Cosme Alvarado-Esquivel, Laboratorio de Investigacion Biomedica, Facultad de Medicina y Nutricion, Avenida Universidad S/N, 34000 Durango, Dgo, Mexico. Email: alvaradocosme@yahoo.com
}

doi: http://dx.doi.org/10.14740/jocmr2131w

\section{Introduction}

The protozoa parasite Entamoeba histolytica is an important cause of morbidity and mortality worldwide $[1,2]$. Infections with E. histolytica are common and are one of the major health problems in developing countries [3, 4]. Humans are the host of $E$. histolytica and there are no other known animal reservoirs of this parasite [5]. The clinical spectrum of $E$. histolytica infections varies from asymptomatic infection to hemorrhagic colitis and extra-intestinal disease [6]. Most persons infected with E. histolytica are carriers [7]. Infection with $E$. histol$y$ tica is responsible from a considerable number of cases of prolonged diarrhea in travelers [8]. In addition, infection with $E$. histolytica may lead to the development of live-threatening abscess in liver, brain [9] or lungs [5]. Transmission of E. histolytica occurs in areas with poor sanitation by contamination of drinking water or food with human feces [10]. Water-associated outbreaks of E. histolytica disease have been reported [11]. Transmission of E. histolytica can also be sexual [12].

Very little is known on the seroepidemiology of E. histolytica infection in rural adults in Mexico. Rural communities in Mexico have commonly poor sanitation, and this is an important condition for transmission of E. histolytica among the population. A considerable number of houses in rural Mexico have poor availability of drinkable water and poor disposal of excretes. Therefore, contamination of water and food with $E$. histolytica is highly feasible to occur in rural communities. The lack of laboratory tests for diagnosis of E. histolytica infection in rural health centers does not allow having reliable statistical information about the magnitude of $E$. histolytica exposure in rural Mexico. We sought to determine the seroprevalence of $E$. histolytica IgG antibodies in adults in rural Durango, Mexico. Furthermore, socio-demographic and behavioral characteristics of the rural subjects associated with E. histolytica seropositivity were investigated.

\section{Materials and Methods}

\section{Study design and study population}

The design of this study was cross-sectional. We analyzed stored serum samples used in a previous survey about the 
seroepidemiology of Toxoplasma gondii infection in rural populations in Durango, Mexico [13]. Serum samples were collected from December 2006 to August 2007 in three rural communities: San Dimas, Villa Montemorelos, and Santa Clara. Inclusion criteria for enrollment were: 1) inhabitants of rural Durango; 2) aged 18 years and older; 3 ) any sex; and 4) who accepted to participate in the survey. Exclusion criteria for enrollment were: 1) subjects with insufficient amount of serum; and 2) subjects with incomplete socio-demographic and behavioral data. Selection of subjects was performed randomly. In total, 282 subjects were included in this study, 94 of them were inhabitants of San Dimas; 82 were inhabitants of Villa Montemorelos, and 106 were inhabitants of Santa Clara.

\section{General socio-demographic and behavioral characteris- tics of rural adults}

Socio-demographic and behavioral characteristics of the participants were obtained with the aid of a standardized questionnaire. Socio-demographic items included age, birthplace, residence, educational level, socio-economic status, and employment. Housing conditions of the participants were determined by using the Bronfman's criteria [14]. This tool allowed to assess crowding, type of flooring (ceramic, concrete, soil), availability of drinkable water (within the house, out of the house), and form of elimination of excretes (flush toilet, latrine, or other). In addition, the educational level (years of education) of the head of the family was recorded. Behavioral items included consumption of unpasteurized milk or untreated water, consumption of unwashed raw vegetables or fruits, frequency of eating away from home (in restaurants or fast food outlets), raising farm animals, foreign travel, and contact with soil (gardening or agriculture).

\section{Laboratory tests}

Serum samples of the participants were analyzed for anti-E. histolytica IgG antibodies by a commercially available enzyme immunoassay "E. histolytica IgG (Amebiasis) ELISA" kit (Diagnostic Automation Inc., Calabasas, CA). All assays were performed following the manufacturer's instructions. Samples were run along with positive and negative controls in each assay. According to the information included in the kit's insert, the enzyme immunoassay used has a sensitivity of $92 \%$ and a specificity of $100 \%$.

\section{Statistical analysis}

We used the software Epi Info version 7 and SPSS version 15.0 to perform the statistical analysis. For calculation of the sample size, a reference seroprevalence of $4.49 \%$ [15] as the expected frequency for the factor under study, 300,000 as the population size from which the sample was selected, $2.5 \%$ confidence limits, and a $95 \%$ confidence level $(\mathrm{CI})$ were considered. The result of the sample size calculation was 263 subjects. The Pearson's Chi-squared test and the Fisher exact test (when values were small) were used for initial comparison of frequencies among groups. Socio-demographic characteristics, housing conditions, and behavioral variables with a $\mathrm{P}$ value equal to or less than 0.05 obtained in the bivariate analysis were further analyzed by multivariate analysis to determine their association with E. histolytica seropositivity. Odds ratios (OR) and 95\% CIs were calculated by using logistic regression analysis with the Enter method. The HosmerLemeshow goodness of fit test was used to assess the fitness of our regression model. Statistical significance was set at $\mathrm{P}$ value $<0.05$.

\section{Ethical aspects}

Only archival serum samples and data from a previous study [13] were used in the present study. The ethical committee of the Mexican Social Security Institute in Durango City, Mexico approved this previous survey. The purpose and procedures of the study were explained to all participants, and a written informed consent was obtained from all of them.

\section{Results}

Most participants were female (78.0\%), of low socioeconomic status $(77.3 \%)$, and unemployed $(76.6 \%)$. Mean age of participants was $42.91 \pm 17.53$ years old (range $18-91$ years). One hundred and eighteen (41.8\%) of the 282 rural subjects had anti-E. histolytica IgG antibodies. A correlation of E. histolytica seropositivity and socio-demographic and behavioral characteristics and housing conditions of the rural subjects studied is shown in Table 1. Of the socio-demographic data, housing conditions, and behavioral characteristics assessed, the variables age, community of residence, education, consumption of unpasteurized milk, source of drinking water, crowding at home, and educational level of the head of the family had $\mathrm{P}$ values $<0.05$ by bivariate analysis. Other socio-demographic data, housing conditions, and behavioral characteristics including occupation, socio-economic status, type of flooring at home, form of elimination of excretes, foreign travel, raising animals, consumption of untreated water, unwashed raw vegetables or fruits, eating away from home and contact with soil had P values $>0.05$ by bivariate analysis. Further analysis using logistic regression of the socio-demographic, housing conditions, and behavioral characteristics of rural adults showed that $E$. histolytica exposure was positively associated with source of drinking water $(\mathrm{OR}=2.73 ; 95 \% \mathrm{CI}: 1.33-5.58 ; \mathrm{P}=0.005)$, and poor education of the head of the family $(\mathrm{OR}=1.53 ; 95 \% \mathrm{CI}$ : 1.03 - 2.27; $\mathrm{P}=0.03$ ) (Table 2). In contrast, logistic regression analysis showed that E. histolytica exposure was negatively associated with consumption of unpasteurized cow milk (OR $=0.55 ; 95 \% \mathrm{CI}: 0.31-0.96 ; \mathrm{P}=0.03)$, and crowding at home $(\mathrm{OR}=0.33 ; 95 \% \mathrm{CI}: 0.17-0.64 ; \mathrm{P}=0.0009)$. The result of the Hosmer-Lemeshow test $(\mathrm{P}=0.60)$ suggested a good fit of our regression model. 
Table 1. Bivariate Analysis of a Selection of Exposure Variables and Seroprevalence of E. histolytica in General Population in Rural Durango

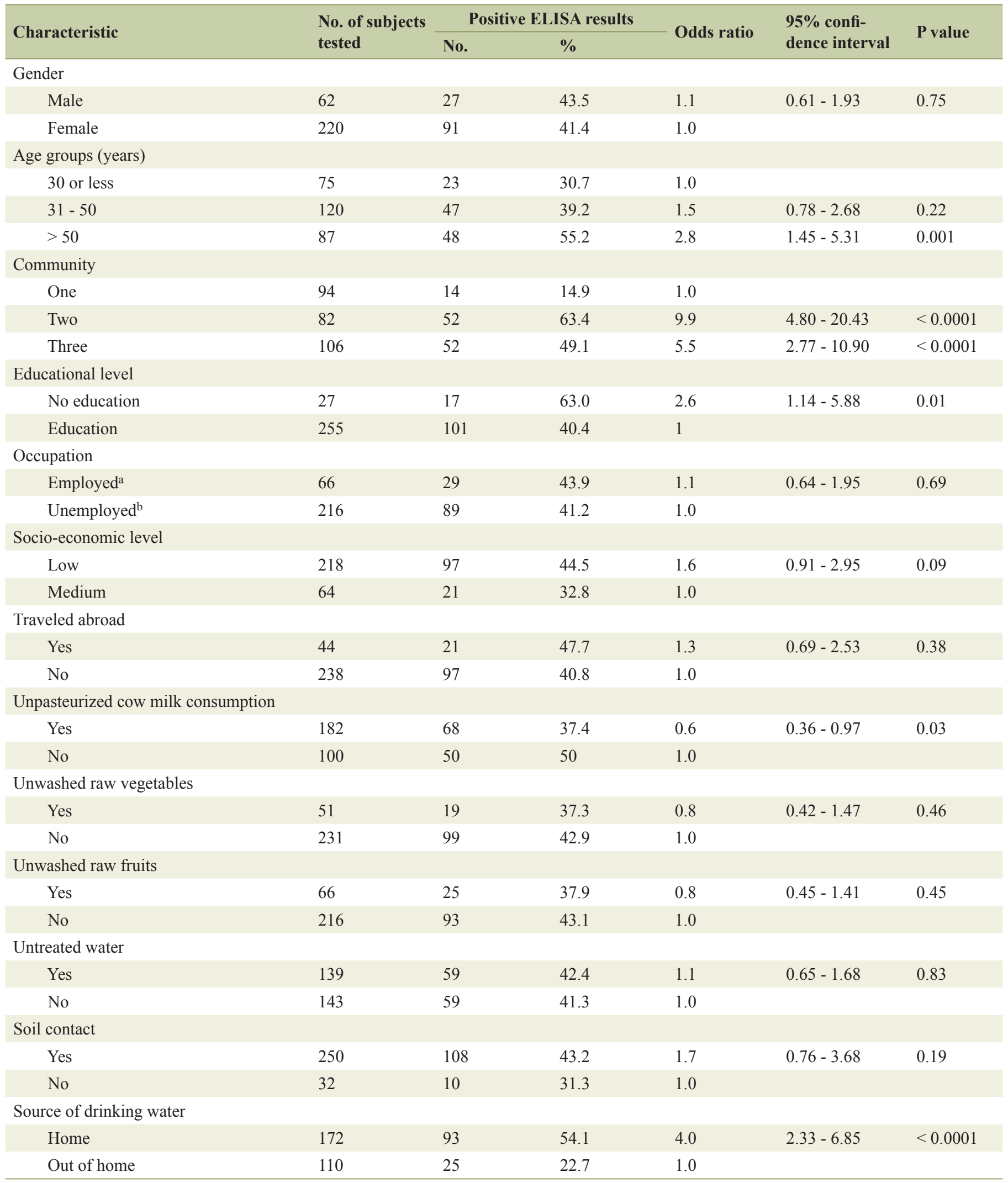


Table 1. Bivariate Analysis of a Selection of Exposure Variables and Seroprevalence of E. histolytica in General Population in Rural Durango - (Continued)

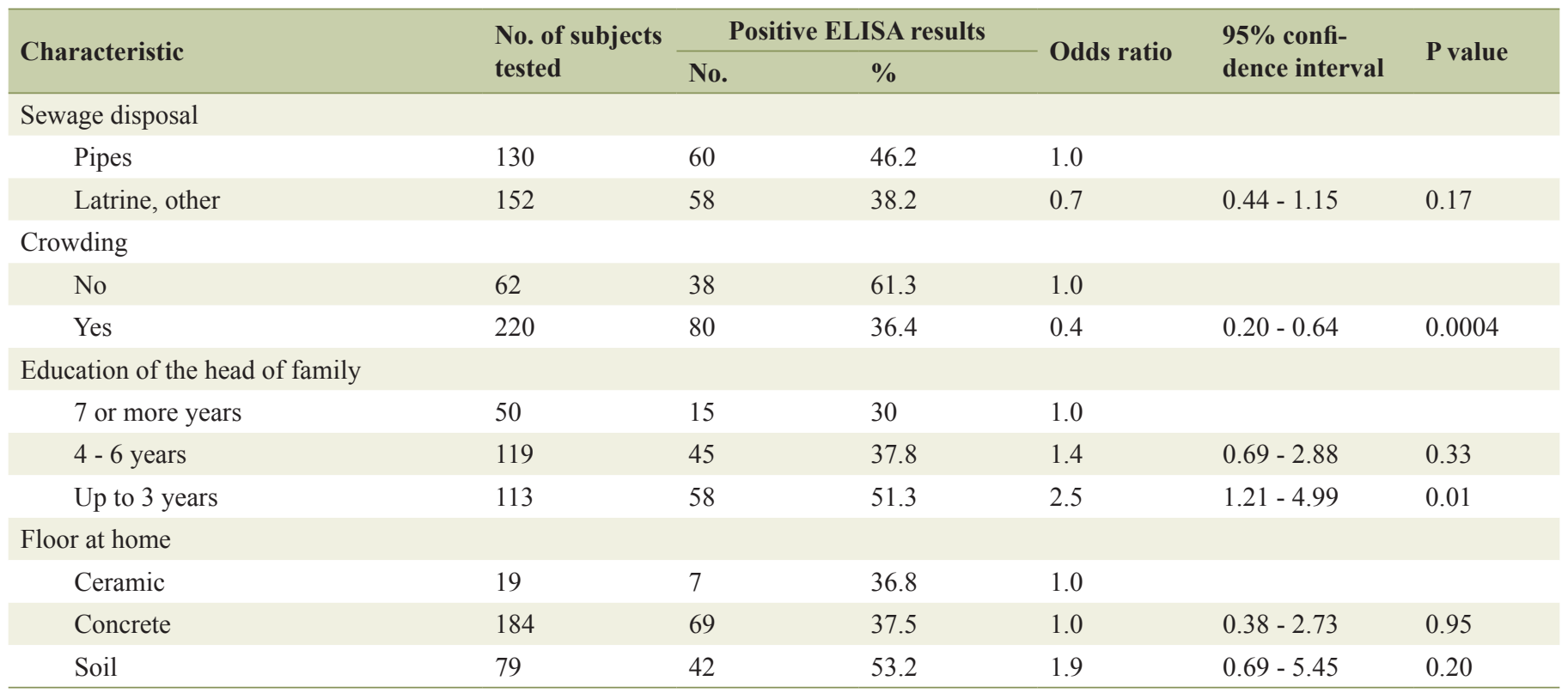

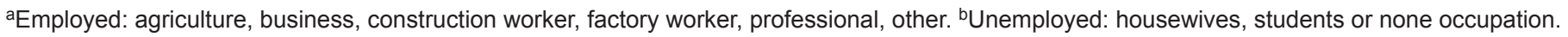

\section{Discussion}

The seroepidemiology of $E$. histolytica infection in rural Mexico has been scantily studied. Although amebiasis has been recognized as a major health problem in Mexico for many years $[16,17]$, very little is known about the seroprevalence of $E$. histolytica infection and risk factors associated with this infection in Mexican populations. Therefore, this study was performed to know the frequency of E. histolytica exposure among adults living in rural areas in the northern Mexican state of Durango. We found an overall $41.8 \%$ seroprevalence of $E$. histolytica infection in adult people of the three rural communities studied. This E. histolytica seroprevalence is higher than other E. histolytica seroprevalences in Mexican populations reported so far. In a previous study in people living in northern Mexican states, researchers found a low $(<5 \%)$ seroprevalence of $E$. histol$y$ tica in all states surveyed [18]. Nearly 25 years have passed between these studies and difference in the seroprevalences might suggest an increase in E. histolytica exposure. However, different laboratory methods were used among the studies; in the previous study, a homemade ELISA was used, whereas we used a commercially available ELISA. The sensitivity and specificity of the homemade ELISA were $95 \%$ and $90.7 \%$, respectively [18]. According to the manufacturer of the commercially available ELISA used in the present study, the assay has a sensitivity and specificity of $92 \%$ and $100 \%$, respectively. The seroprevalence found in adults in rural Durango is also higher than the mean $8.41 \%$ seroprevalence in 32 federal entities and $\leq 8 \%$ in northern states reported in a national survey [17]. However, the presence of antibodies against E. histolytica in the national survey was detected by an indirect hemagglutination test, which is also a different method from the one we used. The seroprevalence of E. histolytica found in our study is also higher than the $4.49 \%$ seroprevalence reported in a second national seroepidemiology survey of E. histolytica infection by using an ELISA [15]. The seroprevalence found in rural Durango is also higher than the $13.8 \%$ prevalence of $E$. histolytica infection in a rural community in the central Mexican state of Morelos obtained by polymerase chain reaction in

Table 2. Results of the Multivariate Regression Analysis

\begin{tabular}{llll}
\hline Variable & P value & Odds ratio & 95\% confidence interval \\
\hline Age & 0.25 & 1.24 & $0.85-1.82$ \\
Community & 0.13 & 1.37 & $0.90-2.08$ \\
No education & 0.37 & 1.54 & $0.58-4.04$ \\
Consumption of raw cow milk & 0.03 & 0.55 & $0.31-0.96$ \\
Water at home & 0.005 & 2.73 & $1.33-5.58$ \\
Crowding & 0.0009 & 0.33 & $0.17-0.64$ \\
Education of the head of family & 0.03 & 1.53 & $1.03-2.27$ \\
\hline
\end{tabular}


stools [19]. However, comparison of the seroprevalence of $E$. histolytica infection with the prevalence of infection based on polymerase chain reaction in stools should be interpreted with care since a poor correlation between intestinal infection and anti-amebic antibody levels has been reported [19]. It is not clear why subjects in rural Durango have a much higher seroprevalence of $E$. histolytica exposure than other populations in Mexico. We searched for potential risk factors associated with E. histolytica in rural Durango. Multivariate analysis showed that E. histolytica exposure was positively associated with source of drinking water and poor education of the head of the family. Subjects with water supply within their home had a higher seroprevalence of $E$. histolytica infection than subjects who obtained water from outside their homes. This finding suggests that $E$. histolytica infection was acquired at home by drinking contaminated water from the public water supplying systems. In Mexico, water supplied by pipes to houses from public water wells is not fully potable. However, many people drink such water in spite of the risk for acquiring infectious diseases. This wrong practice may reflect poor education. In fact, E. histolytica exposure was associated with poor education of the head of the family in our study. In contrast, the negative associations of $E$. histolytica exposure with consumption of unpasteurized cow milk and crowding at home found in the present study suggest that these characteristics did not play any important role in E. histolytica infection among the subjects studied.

\section{Conclusions}

We concluded that the seroprevalence of infection with $E$. histolytica found in rural populations in Durango is higher than $E$. histolytica seroprevalences reported in other Mexican populations. The correlates of E. histolytica seropositivity found in the present study can be used for an optimal planning of preventive measures against $E$. histolytica infection.

\section{Conflict of Interest}

None.

\section{References}

1. van Hal SJ, Stark DJ, Fotedar R, Marriott D, Ellis JT, Harkness JL. Amoebiasis: current status in Australia. Med J Aust. 2007;186(8):412-416.

2. Choudhuri G, Rangan M. Amebic infection in humans. Indian J Gastroenterol. 2012;31(4):153-162.

3. Mortimer L, Chadee K. The immunopathogenesis of Entamoeba histolytica. Exp Parasitol. 2010;126(3):366-380.

4. Ralston KS, Petri WA, Jr. Tissue destruction and in- vasion by Entamoeba histolytica. Trends Parasitol. 2011;27(6):254-263.

5. Tengku SA, Norhayati M. Public health and clinical importance of amoebiasis in Malaysia: a review. Trop Biomed. 2011;28(2):194-222.

6. Chacin-Bonilla L. [An update on amebiasis]. Rev Med Chil. 2013;141(5):609-615.

7. Nair GV, Variyam EP. Noninvasive intestinal amebiasis: Entamoeba histolytica colonization without invasion. Curr Opin Infect Dis. 2014;27(5):465-469.

8. Slack A. Parasitic causes of prolonged diarrhoea in travellers - diagnosis and management. Aust Fam Physician. 2012;41(10):782-786.

9. Petri WA, Haque R. Entamoeba histolytica brain abscess. Handb Clin Neurol. 2013;114:147-152.

10. Marie C, Petri WA, Jr. Amoebic dysentery. BMJ Clin Evid. 2013;2013

11. Karanis P, Kourenti C, Smith H. Waterborne transmission of protozoan parasites: a worldwide review of outbreaks and lessons learnt. J Water Health. 2007;5(1):1-38.

12. Showler AJ, Boggild AK. Entamoeba histolytica. CMAJ. 2013;185(12):1064.

13. Alvarado-Esquivel C, Cruz-Magallanes HM, EsquivelCruz R, Estrada-Martinez S, Rivas-Gonzalez M, Liesenfeld O, Martinez-Garcia SA, et al. Seroepidemiology of Toxoplasma gondii infection in human adults from three rural communities in Durango State, Mexico. J Parasitol. 2008;94(4):811-816.

14. Bronfman M, Guiscafre H, Castro V, Castro R, Gutierrez $\mathrm{G}$. [Strategies for improving the therapeutic patterns used in acute diarrhea in primary medical care units. II. The measurement of inequality: a methodologic strategy, analysis of the socioeconomic features of the sample]. Arch Invest Med (Mex). 1988;19(4):351-360.

15. Gonzalez CR, Isibasi A, Ortiz-Navarrete V, Paniagua J, Garcia JA, Ramirez A, Salvatierra B, et al. Prevalence of antibodies against Entamoeba histolytica in Mexico measured by ELISA. Epidemiol Infect. 1995;115(3):535543.

16. Sepulveda B. Amebiasis: host-pathogen biology. Rev Infect Dis. 1982;4(6):1247-1253.

17. Caballero-Salcedo A, Viveros-Rogel M, Salvatierra B, Tapia-Conyer R, Sepulveda-Amor J, Gutierrez G, OrtizOrtiz L. Seroepidemiology of amebiasis in Mexico. Am J Trop Med Hyg. 1994;50(4):412-419.

18. Isibasi A, Gonzalez C, Ortiz V, Muy M, Paniagua J, Blanco F, Pelayo R, et al. [Seroepidemiology of amebiasis in the northern region of the Republic of Mexico]. Arch Invest Med (Mex). 1990;21(Suppl 1):163-174.

19. Ramos F, Moran P, Gonzalez E, Garcia G, Ramiro M, Gomez A, C DELM, et al. High prevalence rate of Entamoeba histolytica asymptomatic infection in a rural Mexican community. Am J Trop Med Hyg. 2005;73(1):8791. 